\title{
Isomorphisms of symplectic planes
}

\author{
William M. Kantor* \\ (Communicated by T. Grundhöfer)
}

\begin{abstract}
Every nondesarguesian symplectic spread is also symplectic over its kernel. Any equivalence of nondesarguesian symplectic spreads preserves the resulting symplectic structures over the kernels.
\end{abstract}

2000 Mathematics Subject Classification. Primary 51A35, 51A40; Secondary 51A50

\section{Introduction}

A spread of a $2 n$-dimensional vector space $V$ is a set $\Sigma$ of $n$-dimensional subspaces such that each nonzero vector is in one and only one member of $\Sigma$. A spread determines an affine plane $\mathfrak{A}(\Sigma)$, whose points are the vectors and whose lines are the translates of the members of $\Sigma$. A spread $\Sigma$ of $V$ is called symplectic if there is a nondegenerate alternating bilinear form $($,$) on V$ such that each $X \in \Sigma$ is totally isotropic: $(X, X)=0$. Then $\mathfrak{A}(\Sigma)$ is called a symplectic plane. Finite symplectic planes have been studied, for example, in $[5,2,9,6,7,8,1$, 4]; finite ones in characteristic 2 have properties not shared by planes defined using arbitrary spreads.

The kernel $\mathbf{K}(\mathfrak{A}(\Sigma))$ of the spread $\Sigma$ or plane $\mathfrak{A}(\Sigma)$ is the set of all additive endomorphisms $g$ of $V$ such that $X g \subseteq X$ for all $X \in \Sigma$. This is the largest skew field $\mathbf{K}$ over which $V$ can be viewed as a vector space $V_{\mathbf{K}}$ such that $\Sigma$ consists of subspaces. Note that $\mathfrak{A}(\Sigma)$ and $\Sigma$ are nondesarguesian precisely when $\operatorname{dim} V_{\mathbf{K}}>2$.

Two spreads of $V$ are called equivalent if there is an invertible semilinear transformation sending one to the other. Our goal is to study this notion in the case of symplectic spreads.

Theorem 1. Let $\Sigma_{1}$ and $\Sigma_{2}$ be nondesarguesian symplectic spreads in a $K$-space $V$ equipped with a nondegenerate alternating bilinear form $($,$) . Assume that g \in \Gamma \mathrm{L}(V)$ sends $\Sigma_{1}$ to $\Sigma_{2}$.

\footnotetext{
${ }^{*}$ This research was supported in part by NSF Grant DMS 0242983.
} 
(i) Then $\Sigma_{i}$ is a symplectic spread of the $\mathbf{K}_{i}$-space $V=V_{\mathbf{K}_{i}}$, where the kernel $\mathbf{K}_{i}$ of $\Sigma_{i}$ is commutative $(i=1,2)$.

(ii) Let $(,)_{\mathbf{K}_{i}}$ be a nondegenerate alternating bilinear form on $V_{\mathbf{K}_{i}}$ with respect to which $\Sigma_{i}$ is symplectic. Then $g$ sends $\mathbf{K}_{1}$ to $\mathbf{K}_{2}, V_{\mathbf{K}_{1}}$ to $V_{\mathbf{K}_{2}}$ and preserves the symplectic structure: $(u g, v g)_{\mathbf{K}_{2}}=k_{2}\left[(u, v)_{\mathbf{K}_{1}}\right]^{\sigma}$ for some $k_{2} \in \mathbf{K}_{2}^{*}$, some $\sigma \in$ $\operatorname{Aut}\left(\mathbf{K}_{2}\right)$, and all $u, v \in V$.

This theorem also holds trivially when the spreads are desarguesian and the kernels are fields (cf. Section 4).

For the situation in (ii), in [5, Theorem 3.5] we asked whether there is some $h \in \mathbf{K}_{2}^{*}$ such that $g h^{-1}$ preserves the symplectic geometry over the original field $K$. In general we see no reason why this should occur, even when $V$ is finite, but it does hold under suitable numerical assumptions:

Theorem 2. Let $\Sigma_{1}$ and $\Sigma_{2}$ be spreads in a finite $K$-space $V$ that are symplectic with respect to the nondegenerate alternating bilinear form ( , ). Assume that $g \in \Gamma \mathrm{L}(V)$ sends $\Sigma_{1}$ to $\Sigma_{2}$. If $\mathbf{K}_{2}$ is the kernel of $\Sigma_{2}$, and if either $|K|$ is even or $\left[\mathbf{K}_{2}: K\right]$ is odd, then $g=s k_{2}$ with $k_{2} \in \mathbf{K}_{2}^{*}$ and $s \in \Gamma \mathrm{L}(V)$ satisfying $(u s, v s)=k(u, v)^{\sigma}$ for some $k \in K^{*}$, some $\sigma \in \operatorname{Aut}(K)$, and all $u, v \in V$.

Versions of the last theorem were proved in [5, Theorem 3.5] and [9], assuming that $|K|$ is even or $\operatorname{dim} V=4$, respectively. In practice, "usually" $\mathbf{K}_{2}=K$, in which case the theorem is a special case of Theorem 1.

The elementary proofs of the above results use the associated symplectic polarities.

\section{Background}

We refer to [3] for the standard background concerning spreads, translation planes and their kernels. For example, any isomorphism $\mathfrak{A}\left(\Sigma_{1}\right) \rightarrow \mathfrak{A}\left(\Sigma_{2}\right)$ that sends 0 to 0 is induced by a semilinear transformation sending $\Sigma_{1}$ to $\Sigma_{2}$. We refer to [10] for background concerning symplectic geometry. If $($,$) is a nondegenerate alternating bilinear form on V$, then $\Gamma \operatorname{Sp}(V)=\left\{g \in \Gamma \mathrm{L}(V) \mid(u g, v g)=k(u, v)^{\sigma}\right.$ for some $k \in K^{*}$ and $\sigma \in \operatorname{Aut}(K)$, and all $u, v \in V\}$ (cf. the statements of both of the above theorems). Associated with (, ) there is a symplectic polarity $\theta: W \rightarrow W^{\theta}=\{v \in V \mid(v, W)=0\}$ of the projective geometry $\mathrm{PG}(V)$ of $V$, and the corresponding centralizer $C_{\mathrm{P} \Gamma \mathrm{L}(V)}(\theta)=\operatorname{P\Gamma Sp}(V)$ is $\Gamma \operatorname{Sp}(V)$ modulo scalars.

\section{Uniqueness}

Before proving the theorems, we record a very simple observation (implicit in [5, Theorem 3.5] and reappearing in Section 5):

Proposition 1. Suppose that $\Sigma$ is a spread in a $K$-space $V$, where $K$ is the kernel of $\Sigma$. Then $\Sigma$ is symplectic with respect to at most one symplectic polarity of $V$. 
Proof. If $\Sigma$ is symplectic with respect to the symplectic polarities $\theta$ and $\theta^{\prime}$, then $\theta$ and $\theta^{\prime}$ send each member of $\Sigma$ to itself. Hence, so does $\theta \theta^{\prime}$. The collineation $\theta \theta^{\prime}$ of $\operatorname{PG}(V)$ is induced by a semilinear transformation that fixes every member of $\Sigma$ and so is in the kernel $K$. Then $\theta \theta^{\prime}$ fixes every member of $\operatorname{PG}(V)$, and hence $\theta \theta^{\prime}=1$.

In general, if $\mathbf{K}$ is the kernel of $\Sigma$ then $\theta^{\prime}$ lies in the $\operatorname{coset} \theta\left(\mathbf{K}^{*} / K^{*}\right)$.

\section{$4 \quad$ Lifting symplectic structures}

We will be viewing a vector space $V$ over more than one skew field. As in the statement of Theorem 1, if one of these skew fields is called $F$, and we wish to emphasize that we are viewing $V$ as an $F$-space, we will write $V_{F}$ instead of $V$.

Theorem 3. Let $\Sigma$ be a nondesarguesian symplectic spread of the $K$-space $V$, with ker$n e l \mathbf{K} \supseteq K$. Then $\mathbf{K}$ is commutative, and there is a nondegenerate alternating bilinear form $(,)_{\mathbf{K}}$ on $V_{\mathbf{K}}$ such that

(i) $\Sigma$ is a symplectic spread of $V_{\mathbf{K}}$ with respect to $(,)_{\mathbf{K}}$,

(ii) $f\left((,)_{\mathbf{K}}\right)$ is the given nondegenerate alternating bilinear form on $V$ for some nonzero $K$-linear functional $f: \mathbf{K} \rightarrow K$, and

(iii) $(,)_{\mathbf{K}}$ and $f$ are uniquely determined up to multiplication by elements of $\mathbf{K}^{*}$ and $K^{*}$, respectively.

Proof. (i) Let $G$ denote the group of all automorphisms and all anti-automorphisms of $\operatorname{PG}(V)$ that fix each member of $\Sigma$. Then $G$ contains the given polarity $\theta$ and has a normal subgroup $\mathbf{K}^{*} / K^{*}$ consisting of the elements of $\operatorname{P\Gamma L}(V)$ that induce the identity on $\Sigma$. It follows that $\theta$ permutes the set of $\mathbf{K}$-invariant subspaces of $V$; these are precisely the subspaces of $V_{\mathbf{K}}$. Consequently, by restricting $\theta$ to the subspaces of $V_{\mathbf{K}}$ we obtain a polarity $\theta_{\mathbf{K}}$ of $\mathrm{PG}\left(V_{\mathbf{K}}\right)$. Clearly, $\Sigma$ is still a spread of $V_{\mathbf{K}}$, while each member of $\Sigma$ is still sent to itself by $\theta_{\mathbf{K}}$. Each point of $\mathrm{PG}\left(V_{\mathbf{K}}\right)$ lies in some member of $\Sigma$ and hence is perpendicular to itself. Thus, since we are assuming that $\operatorname{dim} V_{\mathbf{K}}>2$, by [10, p. 53] $\mathbf{K}$ is commutative and $\theta_{\mathbf{K}}$ is a symplectic polarity determined by a nondegenerate alternating bilinear form $(,)_{\mathbf{K}}$ on $V_{\mathbf{K}}$, as required.

(ii) If $W$ is any $\mathbf{K}$-subspace then $W \theta_{\mathbf{K}}=W \theta$. Hence, if $u, v \in V$ and $(u, v)_{\mathbf{K}}=0$ then $(u, v)=0$. For fixed $u \neq 0$ it follows that $f_{u}\left((u, v)_{\mathbf{K}}\right):=(u, v)$ is a well-defined map $f_{u}: \mathbf{K} \rightarrow K$, and hence is a $K$-linear functional. Similarly, any two such maps $f_{u}$ agree on each $v \in V$. This implies (ii).

(iii) Each symplectic polarity arises from an alternating bilinear form that is uniquely determined up to a scalar.

This result and Theorem 1 also hold in the desarguesian case if $\mathbf{K}$ is commutative. When $\mathbf{K}$ is noncommutative, there is no symplectic structure possible on $V_{\mathbf{K}}$, but it is not clear whether or not, in this case, a desarguesian spread can be symplectic when viewed over a subfield of $\mathbf{K}$; it is also not clear that this is even an interesting question. 
Proof of Theorem 1. Theorem 1(i) and Theorem 3(i) are the same. For Theorem 1(ii), observe that the uniqueness part of Theorem 3(iii) implies that $g$ must send the form $(,)_{\mathbf{K}_{1}}$ to $(,)_{\mathbf{K}_{2}}$ up to a scalar and a field automorphism (since $g$ is semilinear but not necessarily linear).

\section{Proof of Theorem 2}

Let $\theta$ be the symplectic polarity of $\mathrm{PG}(V)$ associated with (, , ); it is the identity on both $\Sigma_{1}$ and $\Sigma_{2}$. Then $\theta$ and $\theta^{g}=g^{-1} \theta g$ are symplectic polarities each of which is the identity on $\Sigma_{2}$. It follows that $\theta \theta^{g}$ arises from an element of $\Gamma \mathrm{L}(V)$ that is the identity on $\Sigma_{2}$, and hence lies in $\mathbf{K}_{2}$. Since $\left(\left|\mathbf{K}_{2}\right|-1\right) /(|K|-1)$ is odd by hypothesis, $\theta \theta^{g}$ is an element of $\operatorname{P\Gamma L}(V)$ of odd order. Then $\theta^{\bar{h}}=\theta^{g}$ for some $\bar{h}$ in the dihedral group $\left\langle\theta, \theta^{g}\right\rangle$, and hence $\bar{g} \bar{h}^{-1}$ commutes with $\theta$, where $\bar{g}$ is the element of $\operatorname{P\Gamma L}(V)$ induced by $g$. Consequently, $\bar{g} \bar{h}^{-1} \in \operatorname{P\Gamma Sp}(V)$.

As in Proposition 1, $\bar{h}$ is induced by some $h \in \mathbf{K}_{2}^{*}$. Thus, $g=s k h$ with $s \in \Gamma \operatorname{Sp}(V)$ and $k \in K^{*}$, and then $k h \in K h \subseteq \mathbf{K}_{2}$.

Remarks. 1. When $\mathbf{K}_{1}=\mathbf{K}_{2}=K$ the theorem states that $(u s, v s)=k(u, v)^{\sigma}$ for some $k \in K^{*}, \sigma \in \operatorname{Aut}(K)$ and all $u, v \in V$; that is, $g \in \Gamma \operatorname{Sp}(V)$. The above argument is simply that $\theta^{g}=\theta$ as in Proposition 1, and hence $g \in \Gamma \operatorname{Sp}(V)$. This is already contained in Theorem 1(ii). For nondesarguesian $\Sigma$, this is exactly the case considered in [9].

2. An immediate consequence of Theorem 2 is that, under the stated numerical hypotheses regarding the kernel, if $\Sigma_{1}$ and $\Sigma_{2}$ are equivalent then some element of $\Gamma \mathrm{L}(V)$ sends $\Sigma_{1}$ to $\Sigma_{2}$ while preserving the symplectic structure.

Kernels can be nontrivial [5] or difficult [7] to determine. When the characteristic is 2 there is no need to find the kernel in order to use the above consequence; and this was the crucial use made of Theorem 2 in those references. However, it seems doubtful that the preceding consequence holds (for finite $V$ ) without any numerical assumptions.

3. It is natural to ask: how does the above proof differ from the argument in [5, Theorem 3.5]? Here we have used the fact that $\theta \theta^{g}$ lies in the kernel of the spread as a projective transformation of $V$, which enabled some of the problems in the odd characteristic case to disappear. This should have been noticed 20 years ago. It wasn't. Therefore, only the characteristic 2 case was handled at that time.

\section{References}

[1] S. Ball, J. Bamberg, M. Lavrauw, T. Penttila, Symplectic spreads. Des. Codes Cryptogr. 32 (2004), 9-14. MR2072313 (2005c:51005) Zbl 1056.51008

[2] A. R. Calderbank, P. J. Cameron, W. M. Kantor, J. J. Seidel, $Z_{4}$-Kerdock codes, orthogonal spreads, and extremal Euclidean line-sets. Proc. London Math. Soc. (3) 75 (1997), 436-480. MR1455862 (98i:94039) Zbl 0916.94014

[3] P. Dembowski, Finite geometries. Springer 1968. MR0233275 (38 \#1597) Zbl 0159.50001 
[4] N. Johnson, O. Vega, Symplectic spreads and symplectically paired spreads. Note di Matematica, to appear.

[5] W. M. Kantor, Spreads, translation planes and Kerdock sets. I. SIAM J. Algebraic Discrete Methods 3 (1982), 151-165. MR655556 (83m:51013a) Zbl 0493.51008

[6] W. M. Kantor, M. E. Williams, New flag-transitive affine planes of even order. J. Combin. Theory Ser. A 74 (1996), 1-13. MR1383501 (97e:51012) Zbl 0852.51005

[7] W. M. Kantor, M. E. Williams, Symplectic semifield planes and $\mathbb{Z}_{4}$-linear codes. Trans. Amer. Math. Soc. 356 (2004), 895-938. MR1984461 (2005e:51011) Zbl 1038.51003

[8] A. Maschietti, Symplectic translation planes and line ovals. Adv. Geom. 3 (2003), 123-143. MR1967995 (2004c:51008) Zbl 1030.51002

[9] T. Penttila, B. Williams, Ovoids of parabolic spaces. Geom. Dedicata 82 (2000), 1-19. MR1789057 (2001i:51005) Zbl 0969.51008

[10] D. E. Taylor, The geometry of the classical groups. Heldermann 1992. MR1189139 (94d:20028) Zbl 0767.20001

Received 14 June, 2006

W. M. Kantor, University of Oregon, Eugene, OR 97403, USA

Email: kantor@math.uoregon.edu 